TH4B-4

\title{
COMPLEX LEAKY WAVES OF A PARTIALLY OPEN NONRECIPROCAL SLOTLINE ON GYROMAGNETIC SUBSTRATE
}

\author{
Ching-Kuang C. Tzuang, Kuen-Fwu Fuh and Michal Mrozowski* \\ Institute of Communication Engineering, National Chiao Tung University \\ No. 1001, Ta Hsueh Road, Hsinchu, Taiwan, R.O.C. \\ * Department of Electronics, Technical University of Gdansk, Gdansk, Poland
}

\begin{abstract}
The full-wave spectral domain approach is applied to investigate a partially open slotline integrated on multi-dielectric layers containing a ferrite substrate. When frequency decreases from high frequency to low frequency, the bounded modes start to leak in the form of improper forward leaky waves. Then one of the improper forward leaky waves becomes proper backward leaky wave as frequency decreases again. This constitutes a pair of a forward leaky wave and a backward leaky wave that further interact in the mode-coupling way when frequency further decreases. All the data presented have been verified by examining the transverse electromagnetic field patterns.
\end{abstract}

\section{Introduction}

The guided properties of planar or quasi-planar transmission lines with gyromagnetic substrate have been reported in various literatures [1-4]. So far these papers concern primarily the dominant mode $[1,2]$, the higher-order modes $[2,3]$ and the complex modes [4] propagation of the transmission lines. On the other hand, the applications of these transmission lines involve filters, circulators, isolators, scannable antenna, etc., which are integral components of modern electrical communication equipment. It is therefore very desirable to understand in details the guided properties of these transmission lines whenever possible.

This paper presents the first attempt to study the complex waves of a partially open slotline in multidielectric media containing a ferrite substrate, although the full wave analysis does not necessarily restrict itself for the slotline structure. In our particular case study, the improper forward leaky waves, and the proper backward leaky waves can coexist in the same guided structure. The mathematical solutions of the complex propagation constants and the associated physical properties presented in the paper are verified.

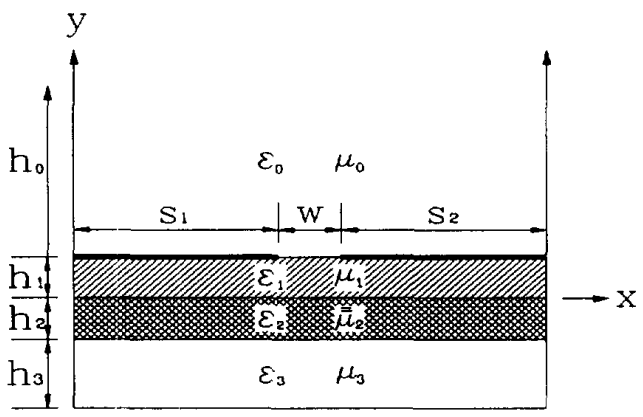

Fig. 1 Cross-sectional geometry of a partially open slotline integrated on the stratified layers containing a ferrite substrate magnetized in $\mathrm{x}$-direction. The structure and material parameters are: $h_{0}=\infty, h_{1}=h_{2}=1 \mathrm{~mm}, h_{3}=1.556 \mathrm{~mm}$, $\mathrm{s}_{1}=\mathrm{s}_{2}=1.628 \mathrm{~mm}, \mathrm{w}=0.3 \mathrm{~mm} \varepsilon_{0 \gamma}=\varepsilon_{3 \gamma}=1.0, \varepsilon_{1 y}=\varepsilon_{2 \gamma}=12.5,4 \pi \mathrm{Ms}$ $=4900 \mathrm{G}$, and $\mathrm{Ho}=30 \mathrm{Oe}$.

\section{Formulation}

Full-wave SDA (spectral domain approach) is used to investigate the slotline structure shown in Fig. 1. The major difference between the SDA incorporated in this paper and the method employed by Geshiro and Itoh [1] is that certain modifications of the formulation are required to satisfy the boundary conditions imposed on the structure shown in Fig. 1, where the top cover is removed. For the bounded waves and the proper backward leaky waves, the transverse fields should vanish at infinity whereas the field amplitude of the improper leaky waves extends to infinity as the field observation point moves to $y=\infty$. The validity of our analysis is checked by two ways. One is for the bounded waves, of which the propagation constants of the dominant modes and the higher 
modes are compared with the avallable data for the closed structures [5,6]. Here, excellent agreement of data for closed and partially open slotlines is achieved. Second, for all those complex waves, with which no existing data can be compared, the transverse field plots are checked against the required boundary conditions in accordance with the various types of complex waves. Parts of these vector field plots will be illustrated in section IV.

\section{Mode Chart of the Partially Open Gyromagnetic Slotline}

The dispersion characteristics of the partially open gyromagnetic slotline as shown in Fig. 1 are plotted in Fig. 2. Dual axes of opposite polarity are employed to illustrate the nonreciprocal guided properties of the waveguide $[5,6]$. The factors $e^{j \omega t}$ and $e^{-j \gamma z}$, where $\gamma=\beta-j \alpha$, are assumed in the analysis. The two real constants, $\beta$ and $-\alpha$, stand for the propagation constant and the attenuation constant (of reverse sign) of the waveguide, which are denoted by solid and dash-dot lines in Fig. 2, respectively. In the 20-to-50 $\mathrm{GHz}$ frequency range of interest, a family of dispersion curves, when read from $50 \mathrm{GHz}$ (right-handside) to $20 \mathrm{GHz}$ (left-hand-side) of Fig. 2, exhibit various types of modes.

In the 44.61-to-50 $\mathrm{GHz}$ range, a pair of modes denoted as F7 and B7 in Fig. 2 represent the forward travelling wave and the backward travelling wave, respectively. They have only real values for $\gamma^{\prime}$ s which are opposite in sign and different in magnitude. The propagation constants for $\mathrm{F} 7$ and $\mathrm{B} 7$ are in very close agreement with those reported in the gyromagnetic finline (closed structure) [5,6]. F7 and B7 represent waves that are bounded in nature, with the transverse fields vanish at infinity. Therefore the fields in the upper air region spread over a finite distance along the positive y direction.

Below the critical point, $\mathrm{f}=44.61(42.39) \mathrm{GHz}$, the bounded wave F7(B7) starts to leak in the form of $\mathrm{TM}_{\mathrm{o}}$ mode under the waveguide dimensions chosen for the design and is denoted as FL7(FL7'). Due to the nonreciprocity, F7 and B7 become leaky at different critical frequency points. Both FL7 and FL7' are the improper forward leaky wave. FL7 (FL7') has positive (negative) values for both $\beta$ and $\alpha$. Both are improper in the sense that the amplitudes of the transverse fields grow exponentially along the ty axis.

As the frequency decreases further and is just below $41.5 \mathrm{GHz}, \mathrm{FL} 7$ wave (read as the number 7 forward leaky wave) becomes BL7, which is a backward leaky wave since $\beta$ is negative and $\alpha$ is posi- tive. The field intensity rises along the negative $\mathrm{z}$ axis while the transverse field amplitude decays along positive $y$ axis (in the direction toward infinity). Thus the backward leaky wave is proper. FL7', however, remains as the forward leaky wave, which is improper.

The BL7 and FL7' finally merge but never contact each other at $f=41.31 \mathrm{GHz}$. Mode coupling, similar to that reported for the generation of complex modes in gyromagnetic finline $[5,6]$, takes place. Below $\mathrm{f}=41.31 \mathrm{GHz}$, the resultant coupled modes remain in the forms of BL7 and FL7' till reaching the lower frequency limit of interest. The rest of the families of the modes show almost the same dispersion characteristics as those discussed above except that the mode coupling may occur for the lower order modes. e.g., FL6 and BL6', at $\beta>0$.

When the bounded waves become leaky, e.g the F7-B7 pair starting to leak as FL7-FL7' pair, the propagation constants $(\beta \mathrm{s})$ and the attenuation constants ( $\alpha s)$ of FL7-FL7' and the rest of the complex waves are different in magnitude and sign. This manifests the facts that this guided structure is nonreciprocal and the leaky waves represent certain loss mechanisms. Therefore the complex propagation constants obtained for the BL7 and FL 7' are no longer complex conjugate pairs because the eigenvalue problem is not hermitian as the case of lossless waveguides [7]. Notice that the complex modes obtained in the lossless gyromagnetic finline are in complex conjugate pairs $[5,6]$, which are in contrast to what we have reported here for the propagation constants of the complex leaky waves.

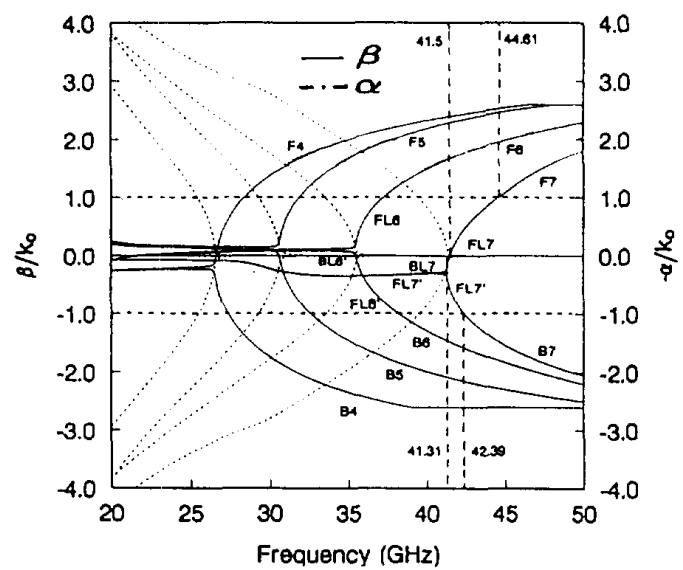

Fig. 2 Dispersion characteristics of the structure shown in Fig. 1 from the 4-th to the 7-th modes. The solid lines and the dash-dot lines are for the real and imaginary parts of the normalized propagation constants, respectively. 


\section{Transverse Fields of the Various Types of waves}

Fig. 3(a) and 3(b) plot the transverse electromagnetic fields for F7 (forward travelling wave). At the conductor surfaces, the tangential electric fields vanish. Along the ty direction, the fields decays. The electromagnetic field singularities around the edges of the slot lines are also observed.

The transverse electric fields of the forward leaky waves FL7 and FL7' are displayed in Fig. 4(a)

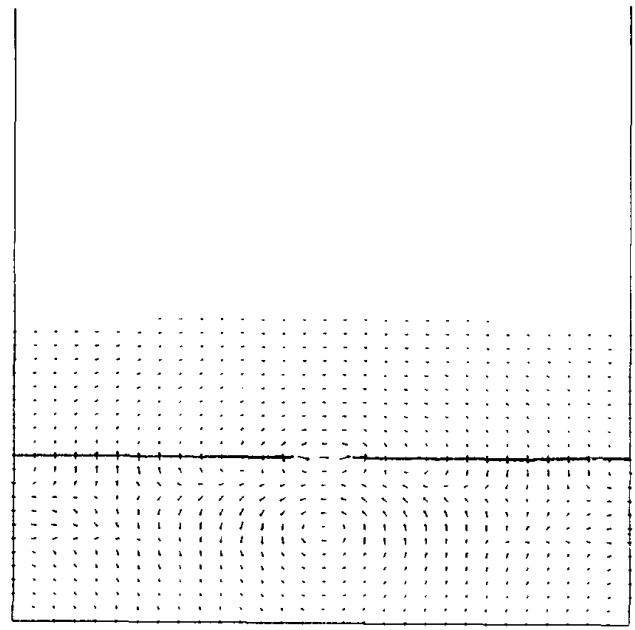

(a)

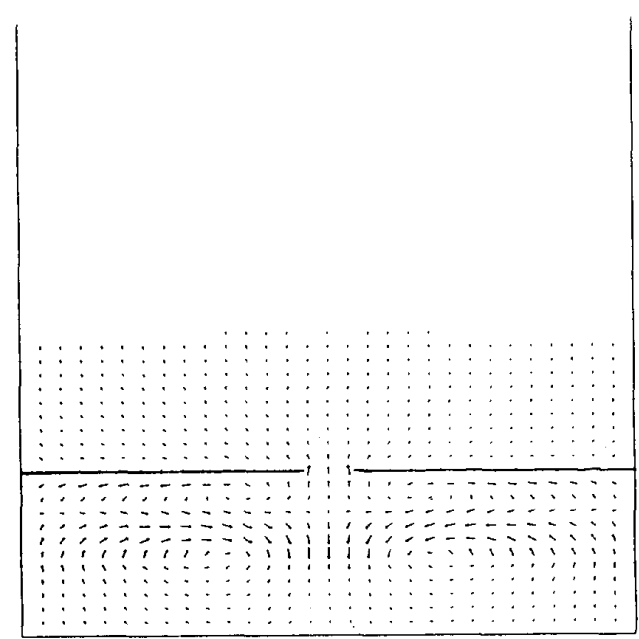

(b)

Fig. 3 Transverse electromagnetic field diagrams of the bounded modes. (a) the transverse electric field of the forward travelling wave F7, (b) the transverse magnetic field of the forward travelling wave F7. All the fields are plotted at $\mathrm{f}=50.0 \mathrm{GHz}$. and Fig. 4(b), respectively. While satisfying the required boundary conditions at all conductor surfaces, the magnified transverse fields in the upper air region exhibit parallel electrical field lines and become perpendicular to the side walls. This implies that the wave does leak in the form of the dominant parallel-plate TM mode (also known as the TEM mode) at an angle $\theta=\cos ^{-1}\left(\beta / k_{0}\right)$ from $z$-axis.

Figs. 5(a) and (b) show the transverse electric field plots of BL7 and FL7' at $40.8 \mathrm{GHz}$, at which the mode-coupling of the two complex waves has already

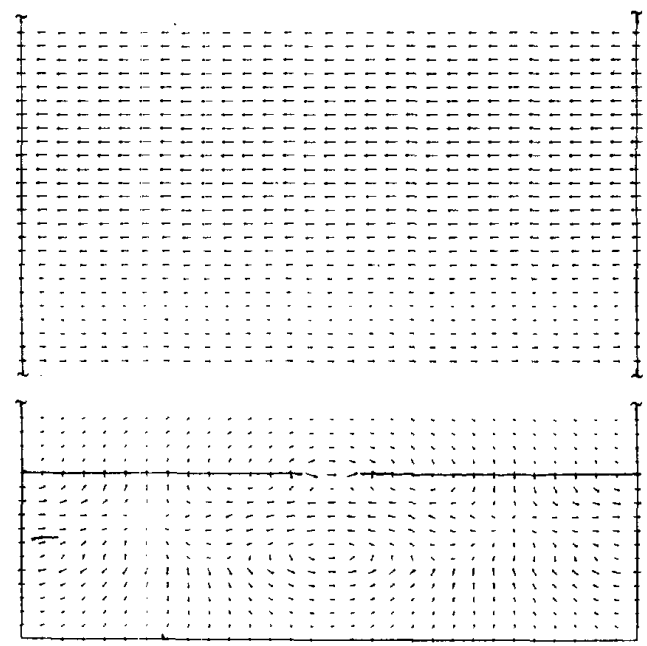

(a)
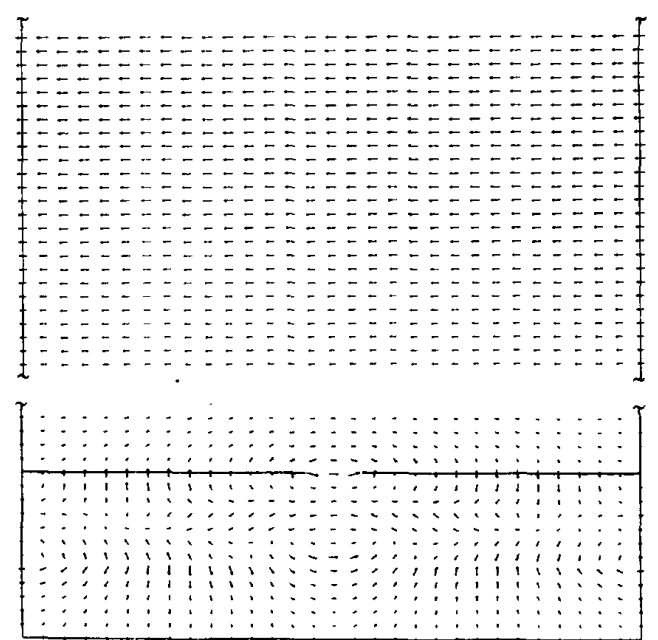

(b)

Fig. 4 Transverse electric field diagrams. (a) the forward leaky wave, $\mathrm{FL7}$, at $\mathrm{f}=42.3 \mathrm{GHz}$, (b) the forward leaky wave, $\mathrm{FL7}^{\prime}$, at $\mathrm{f}=42.3 \mathrm{GHz}$. Field intensity in the upper air region is magnified. 
taken place as discussed in Section III. It is interesting to observe that BL7 rises and FL7' decays along the negative $z$ direction in the same way that a pair of complex modes behave in a closed lossless waveguide. Fig. 5(a) shows a very slowly decaying electric fields that are parallel to each other and perpendicular to the side walls in the upper air region. In contrast, Fig. 5(b) shows very slowly rising electric fields. In both plots the field intensity in the upper air region is magnified.

\section{Conclusion}

Complex leaky waves of a partially open slotline on gyromagnetic substrate have been investigated rigorously using the full-wave spectral domain approach. The data presented in the paper are in good agreement with the available data for the bounded modes and confirmed by the transverse electromagnetic field plots satisfying all the required boundary conditions in accordance with the various types of the leaky waves. The calculated data and field plots show that (1) the gyromagnetic slotline is nonreciprocal, (2) the leaky wave does leak in the form of parallel-plate mode, (3) due to the radiation loss caused by leakage, the complex propagation constants obtained are not in complex conjugate pairs as those reported for the complex modes in closed structures

\section{Acknowledgement}

This work was supported by the National Science Council, Re- public of China, under Grant NSC 82-0404-D009-033 and NSC83-0404-E009-045.

\section{Reference}

[1] M. Geshiro and T. Itoh, "Analysis of double-layered finlines containing a magnetized ferrite," IEEE Trans. Microwave Theory Tech., vol. MTT-35, pp. 1377-1381, Dec. 1987.

[2] J. C. Minor and D. M. Bolle, "Modes in the shielded microstrip on a ferrite substrate transversely magnetized in the plane of the substrate," IEEE Trans. Microwave Theory Tech., vol. MTT-19, pp. 570-577, July 1971.

[3] W. Zieniutycz, "Modes of propagation in slot line with layered substrate containing magnetised ferrite," Electronic Letter, no. 4, vol.19, pp. 135-136, Feb. 1983.

[4] C.-K. C. Tzuang, C.-Y. Lee and J.-T. Kuo, "Complex modes in lossless nonreciprocal finline," Electron Letter, no. 22, vol. 26, pp. 1919-1921, 1990.

[5] C. K. C. Tzuang and J. M. Lin, "Mode-coupling formation of complex modes in a shielded nonreciprocal finline," in 1991 IEEE MTT-S Int. Microwave Symp. Digest, pp.571-574, 1991 .

[6] C. K. C. Tzuang and J. M. Lin, "On the mode-coupling formation of complex modes in nonreciprocal finline," IEEE Trans. Microwave Theory Tech., vol. MTT-41, pp 1400-1408, August 1993

[7] M. Mrozowski, "Waves in shielded lossless isotropic waveguiding structures," $\mathrm{PhD}$ Thesis, Dec. 1989, Technical University of Gdansk, Poland.

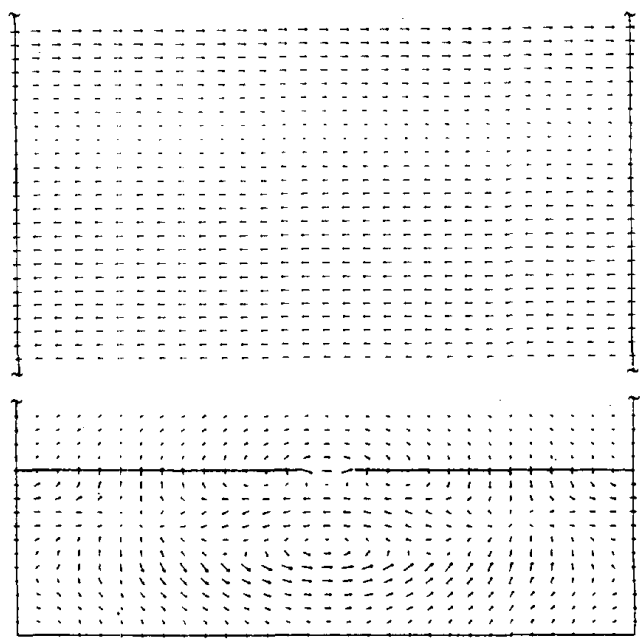

(b)

Fig. 5 Transverse electric field diagrams in the mode-coupling region. (a) the backward leaky wave, BL7, at $f=40.8 \mathrm{GHz}$, (b) the forward leaky wave, FL7', at $\mathrm{f}=40.8 \mathrm{GHz}$. Field intensity in the upper air region is magnified. 